\title{
Genome-Wide Association Studies Reveal the Genetic Basis of Fertility Restoration of CMS-WA and CMS-HL in xian/indica and aus Accessions of Rice (Oryza sativa L.)
}

Pingbo Li, Hao Zhou, Hanyuan Yang, Duo Xia, Rongjia Liu, Ping Sun, Quanxiu Wang, Guanjun Gao, Qinglu Zhang, Gongwei Wang and Yuqing $\mathrm{He}^{*}$ (D)

\begin{abstract}
Background: Wild-abortive cytoplasmic male sterility (CMS-WA) and Honglian CMS (CMS-HL) are the two main CMS types utilized in production of three-line hybrid rice in xian/indica $(X I)$ rice. Dissection of the genetic basis of fertility restoration of CMS-WA and CMS-HL in the core germplasm population would provide valuable gene and material resources for development of three-line hybrid combinations.

Results: In this study, two $F_{1}$ populations with CMS-WA and CMS-HL background respectively were developed using $337 \mathrm{XI}$ and aus accessions being paternal parents. Genome-wide association studies on three fertility-related traits of the two populations for two consecutive years revealed that both fertility restoration of CMS-WA and CMS$\mathrm{HL}$ were controlled by a major locus and several minor loci respectively. The major locus for fertility restoration of CMS-WA was co-located with Rf4, and that for fertility restoration of CMS-HL was co-located with Rf5, which are cloned major restorer of fertility (Rf) genes. Furthermore, haplotype analysis of $R f 4, R f 5$ and $R f 6$, the three cloned major Rf genes, were conducted using the 337 paternal accessions. Four main haplotypes were identified for Rf4, and displayed different subgroup preferences. Two main haplotypes were identified for $R f 5$, and the functional type was carried by the majority of paternal accessions. In addition, eight haplotypes were identified for Rf6.

Conclusions: Haplotype analysis of three $R f$ genes, $R f 4, R f 5$ and $R f 6$, could provide valuable sequence variations that can be utilized in marker-aided selection of corresponding genes in rice breeding. Meanwhile, fertility evaluation of 337 accessions under the background of CMS could provide material resources for development of maintainer lines and restorers.
\end{abstract}

Keywords: GWAS, Rf genes, CMS-WA, CMS-HL, Haplotype analysis, Rice

\section{Background}

Cytoplasmic male sterility (CMS) in higher plants is characterized by the inability to produce functional pollens, and is caused by chimeric open reading frame (ORF) in the mitochondrial genome. Nuclear-encoded restorer of fertility $(R f)$ genes produce proteins that are targeted to mitochondrial and can suppress the function of ORFs conferring CMS (Chen and Liu 2014). The exploitation of

\footnotetext{
* Correspondence: yqhe@mail.hzau.edu.cn

National Key Laboratory of Crop Genetic Improvement and National Center of Crop Molecular Breeding, Huazhong Agricultural University, Wuhan 430070, China
}

CMS and $R f$ genes systems in rice facilitate the development and commercialization of the three-line hybrid rice, which has made tremendous contribution to the food security worldwide ( $\mathrm{Li}$ et al. 2007). According to that the pollen fertility is determined by the sporophytic genotype or gametophytic genotype, CMS could be divided into two types, sporophytic and gametophytic types. Wild-abortive CMS (CMS-WA) and Honglian CMS (CMS-HL) are the two main CMS types utilized in production of three-line hybrid rice in xian/indica (XI) rice, and CMS-WA is a typical sporophytic type while CMS-HL is a gametophytic type (Li et al. 2007). Mining and cloning of $R f$ genes for 
the two CMS types could be of great use in development of three-line hybrid rice combinations, and further improving the yield of rice.

Sterile lines carrying CMS-WA produce pollens that display irregular shape and no starch accumulation (Chen and Liu 2016). CMS-WA is caused by WA352, a novel mitochondrial gene originated recently in wild rice, and the protein WA352 abolishes the function of COX11 in peroxide metabolism, further triggers premature tapetal programmed cell death and finally results in the abortion of pollens (Luo et al. 2013). Previous studies showed that two major genes, $R f 3$ and $R f 4$, could restore the fertility of CMS-WA (Yao et al. 1997, Zhang et al. 1997). Rf4 encodes a pentatricopeptide repeat (PPR) protein with 782 aminoacid residues, which suppresses WA352-mediated male sterility by decreasing WA352 mRNA levels (Kazama and Toriyama 2014, Tang et al. 2014). However, $R f 3$ is still under cloning. In addition, several minor loci conferring fertility restoration of CMS-WA were reported (Bazrkar et al. 2008, Zhuang et al. 2001).

Sterile lines carrying CMS-HL produce pollens that display regular shape and no starch accumulation ( $\mathrm{Hu}$ et al. 2016). CMS-HL is caused by the mitochondrial gene orfH79, and the protein ORFH79 interacts with a subunit of the mitochondrial electron transport chain complex III, further causes energy production dysfunction and oxidative stress, and finally leads to abnormal pollen development (Peng et al. 2010, Wang et al. 2013). Two major genes, $R f 5$ and Rf6, could restore fertility of CMS-HL (Huang et al. 2012a). Rf5 encodes a protein with 16 PPR motifs, which is a component of a restoration of fertility complex conferring the processing of CMS-associated transcript atp6-orfH79, together with at least another two members (Hu et al. 2012, Qin et al. 2016). Similarly, $R f 6$ encodes a protein with 20 PPR motifs, and the protein RF6 forms a new complex with other members to cleave the aberrant transcript atp6-orfH79 (Huang et al. 2015). However, the co-existence of $R f 5$ and $R f 6$ can only restore fertility of $75 \%$ of pollens. In order to further improve pollen fertility and seed-setting rate of $\mathrm{F}_{1}$ lines with CMSHL, novel $R f$ genes are awaited to be identified.

Genome wide association studies (GWAS) have been proved to be powerful in dissection of complex traits in rice (Han and Huang 2013). Up to now, the genetic bases of many important agronomic traits of rice, such as flowering time, grain yield traits, tiller angle, panicle architecture and out-crossing traits, have been investigated with GWAS (Bai et al., 2016, Dong et al. 2016, Guo et al. 2017, Huang et al. 2012b). However, GWAS has not been applied to dissect the genetic basis underlying fertility restoration of CMS-WA and CMS-HL. Therefore, two $F_{1}$ populations with CMS-WA and CMS-HL background respectively were developed using $337 \mathrm{XI}$ and aus accessions being paternal parents in this study, and subjected to evaluation of three fertility-related traits. GWAS of the three traits revealed the corresponding genetic basis. In addition, haplotype analysis of $R f 4, R f 5$ and $R f 6$, the three major genes for CMS-WA and CMS-HL respectively, were conducted.

\section{Results \\ Variation and Correlation of Fertility-Related Traits}

As shown in Fig. 1, after stained with $1 \% \mathrm{I}_{2}-\mathrm{KI}$ solution, the pollens of HUA (Fig. 1a) showed no starch accumulation and were termed completely sterile, and that of the $\mathrm{F}_{1}$ combination 'HUA/Minghui 63' (Fig. 1b) were stained with a color of dark-blue and were termed completely fertile. In contrast, the pollens of YTA (Fig. 1c) showed obvious starch accumulation, although they were completely sterile according to the values of BSS and NSS (data not shown). The pollens of the $F_{1}$ combination 'YTA/9311' (Fig. 1d) were nearly indistinguishable from that of YTA. Thus, the pollen fertility of the $F_{1}$ population with CMS-HL was excluded in this study.

For the population with the CMS-WA background, all the three fertility-related traits displayed continuous and extensive variation (Fig. 2a-c, Additional file 1: Figure S2ac). A significant difference on the distribution of pollen fertility was observed between $F_{1}$ lines with the two $X I$ subgroups being paternal parents (Fig. 2a, Additional file 1: Figure S2a). The values of pollen fertility for the majority of $\mathrm{F}_{1}$ lines with $X I I$ accessions being paternal parents were 0 , while that for the majority of $F_{1}$ lines with $X I I I$ accessions being paternal parents were over $60 \%$. Both the values of BSS and NSS of $\mathrm{F}_{1}$ lines with XI II accessions being paternal parents were obviously higher than that with $X I I$ accessions being paternal parents on average (Fig. 2bc, Additional file 1: Figure S2b-c). All the three traits were significantly positively correlated with each other in year 2013 and 2014, and the highest correlation was observed between pollen fertility and NSS in 2014 with a value of 0.91 (Fig. 3a).

For the population with the CMS-HL background, the two fertility-related traits displayed continuous variation (Fig. 2d-e, Additional file 1: Figure S2d-e). Both the values of BSS and NSS of $F_{1}$ lines with the two XI subgroups accessions being paternal parents were obviously higher than that with aus accessions being paternal parents on average. Both the two traits were significantly positively correlated with each other in year 2013 and 2014, and the highest correlation was observed between BSS and NSS in 2013 with a value of 0.74 (Fig. 3b).

\section{Loci Associated with Fertility-Related Traits}

GWAS for fertility-related traits were conducted with the LMM model for the two $F_{1}$ populations with different CMS background respectively, and a $P$ value of $8.7 \times$ $10^{-8}$ was used as the genome-wide significance thresholds. 
(a)

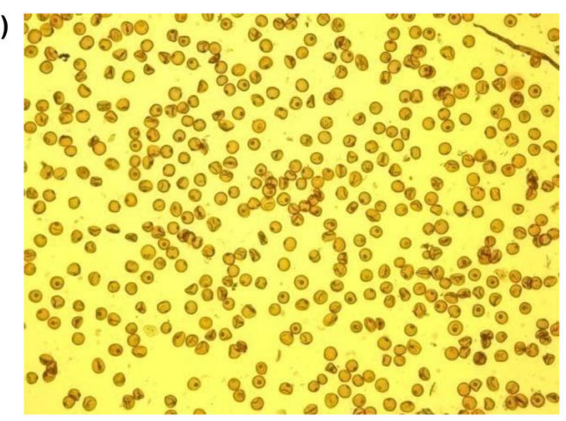

(c)

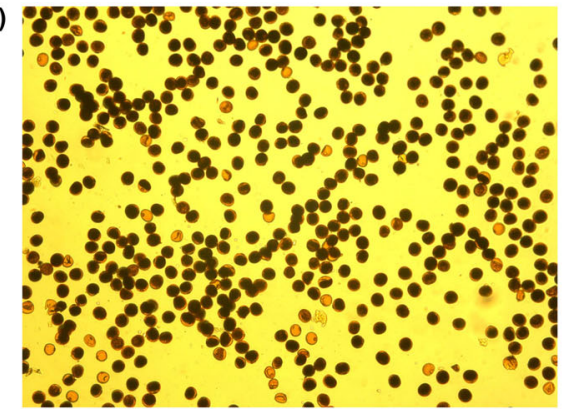

(b)

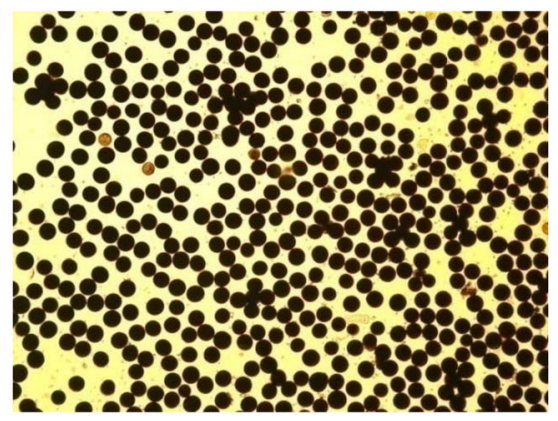

(d)

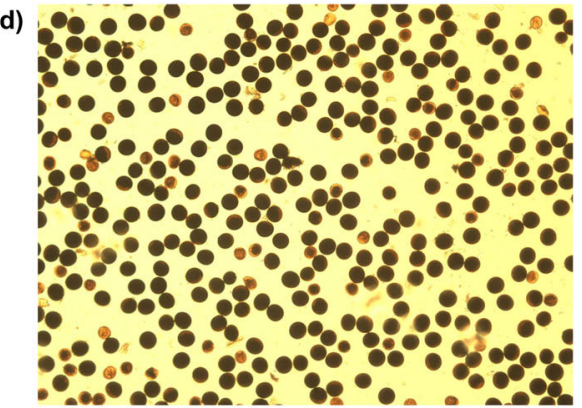

Fig. 1 Pollens of HUA (a), HUA/MH63 (b), YTA (c) and YTA/9311 (d), stained with a 1\% I ${ }_{2}$-KI solution

For the population with CMS-WA background, a total of 3, 8 and 2 loci were detected for pollen fertility, BSS and NSS respectively in 2 years, and the phenotypic variation explained by each locus was ranging from $2.90 \%$ to $48.46 \%$ (Table 1 ). Among those, three major loci around the $18.8 \mathrm{Mb}$ of chromosome 10 were responsible for the three fertility-related traits in both years respectively, which were co-localized with Rf4, the well-known major gene for fertility restoration of CMS-WA
(Table 1, Fig. 4a-c, Additional file 1: Figure S3a-c). The remaining loci were only detected in 1 year.

For the population with CMS-HL background, 5 and 1 loci were detected for BSS and NSS respectively in 2 years, and the phenotypic variation explained by each locus was ranging from $6.35 \%$ to $31.08 \%$ (Table 2). Among those, two major loci around the $18.8 \mathrm{Mb}$ of chromosome 10 were responsible for the two fertility-related traits in both years respectively, which were co-localized with $R f 5$, the well-known major

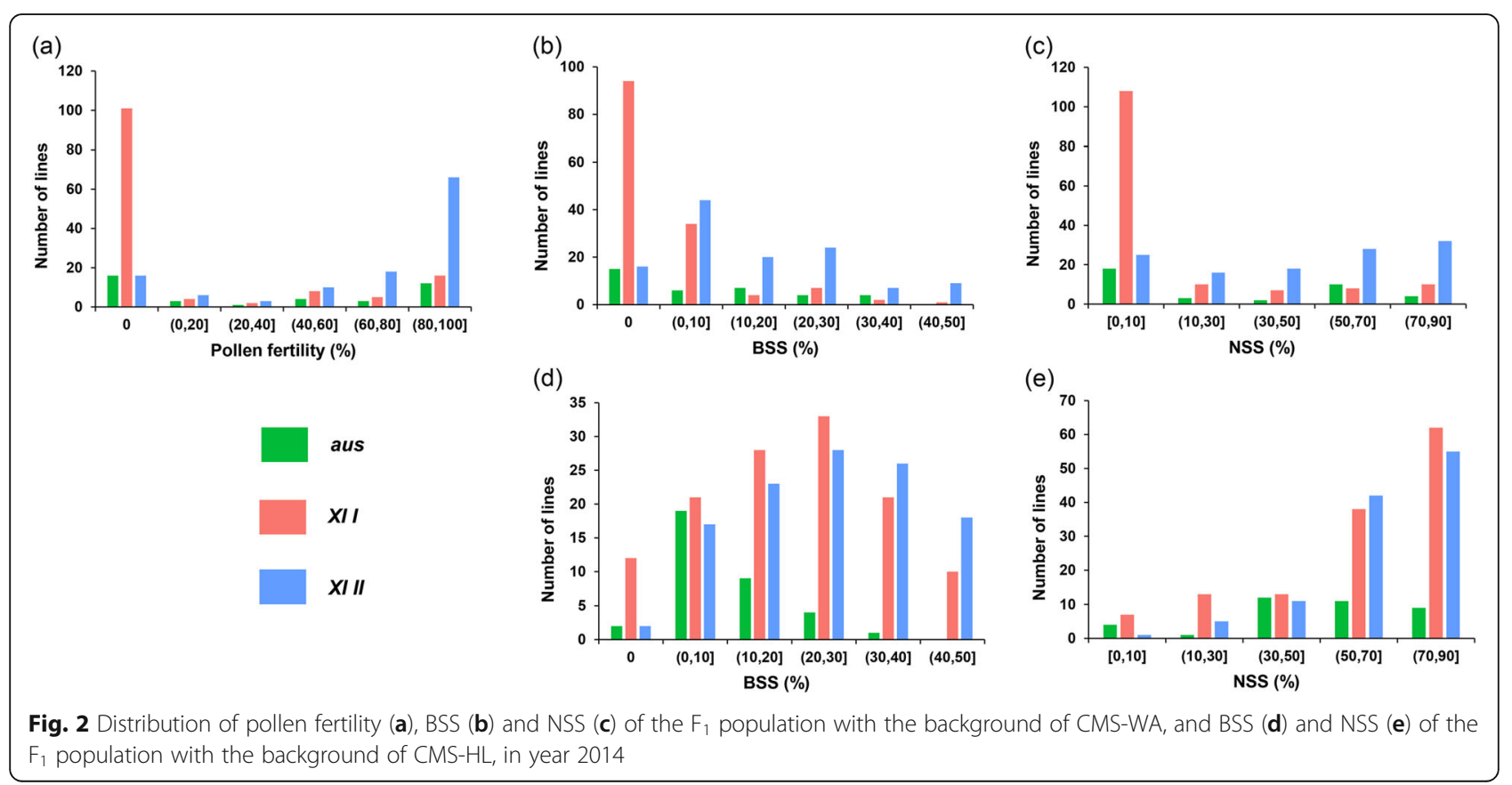




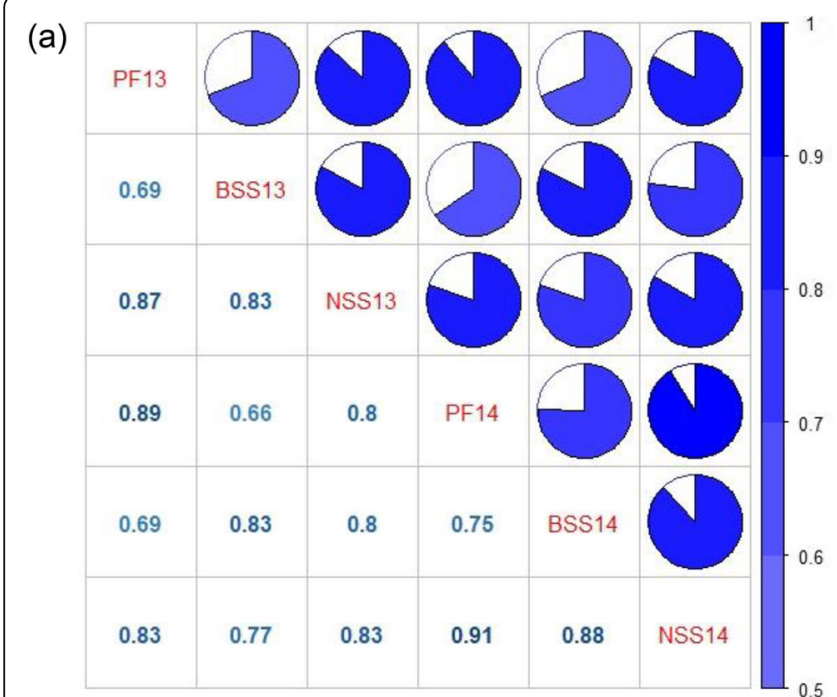

(b)

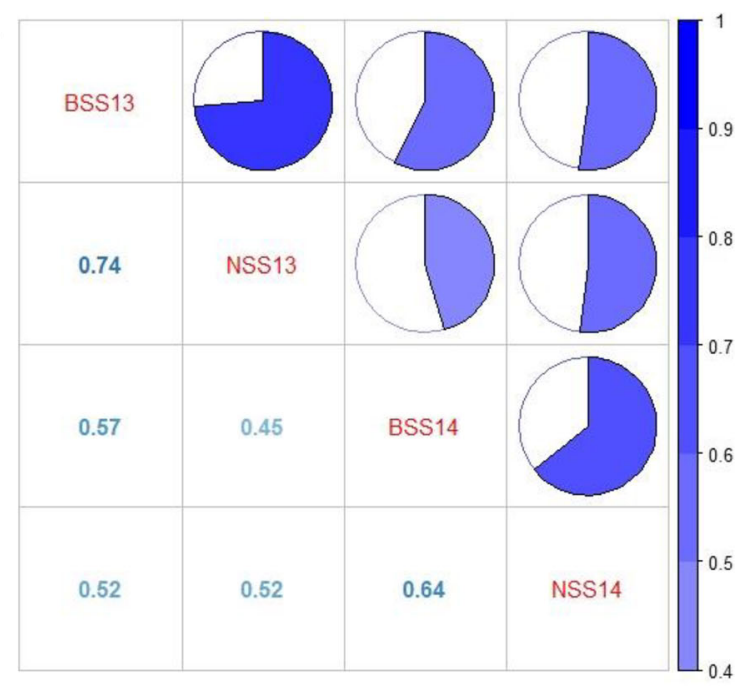

Fig. 3 Correlation coefficients among fertility-related traits in the $F_{1}$ population with the background of CMS-WA (a) and the $F_{1}$ population with the background of CMS-HL (b) in year 2013 and 2014. In $\mathbf{a}$ and $\mathbf{b}$, positive significant correlations were observed in all the traits at the level of $p<0.001$. PF13, BSS13 and NSS13 represent the pollen fertility, BSS and NSS in year 2013 respectively. Similarly, PF14, BSS14 and NSS14 represent the three traits in year 2014 respectively

gene for fertility restoration of CMS-HL (Table 2, Fig. 4d-e, Additional file 1: Figure S3d-e). The remaining loci were only detected in 1 year.

\section{Haplotype Analysis of $R f 4, R f 5$ and $R f 6$}

The co-localization of $R f 4$ and $R f 5$ with the major loci for CMS-WA and CMS-HL detected in this study respectively, indicated that they are likely to be functional genes underlying them. In order to further validate it, haplotype analysis of $R f 4$ and $R f 5$ were performed using the 337 paternal accessions. In addition, $R f 6$, another well-known major gene for CMS-HL, was also subjected to haplotype analysis, though it was not detected in this study.

For Rf4, four main haplotypes and a rare haplotype were classified according to sequence variations in coding region (Fig. 5a, Additional file 2: Table S3). The H1

Table 1 Genome-wide significant associations for pollen fertility, BSS and NSS of the $F_{1}$ population with the background of CMS-WA in year 2013 and 2014 using the linear mixed model

\begin{tabular}{|c|c|c|c|c|c|c|c|c|c|c|c|c|}
\hline \multirow[t]{2}{*}{ Trait } & \multirow[t]{2}{*}{ Chr. } & \multicolumn{5}{|l|}{2013} & \multicolumn{5}{|l|}{2014} & \multirow{2}{*}{$\begin{array}{l}\text { Known } \\
\text { loci }\end{array}$} \\
\hline & & Position & P-LMM & Allele $^{1)}$ & $M^{\prime} F^{2)}$ & PVE $(\%)^{3)}$ & Position & P-LMM & Allele & MAF & $\overline{\text { PVE }(\%)}$ & \\
\hline \multirow[t]{3}{*}{ Pollen fertility } & 1 & & & & & & $5,631,778$ & $2.97 \mathrm{E}-08$ & $A / T$ & 0.168 & 19.01 & $R f 3$ \\
\hline & 9 & & & & & & $10,301,051$ & $8.11 \mathrm{E}-08$ & $\mathrm{G} / \mathrm{A}$ & 0.361 & 2.90 & \\
\hline & 10 & $18,809,352$ & $5.42 \mathrm{E}-12$ & $C / T$ & 0.359 & 37.67 & $18,803,763$ & $2.33 E-20$ & $C / T$ & 0.350 & 48.46 & $R f 4$ \\
\hline \multirow[t]{8}{*}{ BSS } & 4 & 113,551 & $6.54 \mathrm{E}-10$ & $\mathrm{~A} / \mathrm{G}$ & 0.241 & 16.90 & & & & & & \\
\hline & 4 & & & & & & 962,149 & 1.36E-08 & $\mathrm{A} / \mathrm{C}$ & 0.172 & 15.89 & \\
\hline & 6 & $11,935,371$ & $1.18 \mathrm{E}-08$ & T/A & 0.061 & 11.91 & & & & & & \\
\hline & 6 & & & & & & $28,616,342$ & 2.37E-07 & G/A & 0.495 & 2.93 & \\
\hline & 8 & $6,798,111$ & 4.27E-08 & $C / T$ & 0.078 & 25.48 & & & & & & \\
\hline & 9 & & & & & & $10,218,016$ & $3.38 \mathrm{E}-08$ & $G / A$ & 0.358 & 4.45 & \\
\hline & 10 & $18,826,378$ & $3.50 \mathrm{E}-13$ & $\mathrm{~T} / \mathrm{G}$ & 0.176 & 33.54 & $18,803,939$ & $2.02 \mathrm{E}-14$ & $C / T$ & 0.182 & 19.37 & $R f 4$ \\
\hline & 12 & & & & & & $23,866,969$ & 9.55E-09 & $\mathrm{T} / \mathrm{C}$ & 0.325 & 20.10 & \\
\hline \multirow[t]{2}{*}{ NSS } & 4 & 54,458 & 3.07E-08 & $\mathrm{A} / \mathrm{C}$ & 0.326 & 12.51 & & & & & & \\
\hline & 10 & $18,807,815$ & $4.20 \mathrm{E}-10$ & $C / G$ & 0.413 & 41.52 & $18,803,939$ & $2.02 \mathrm{E}-16$ & $\mathrm{C} / \mathrm{T}$ & 0.182 & 19.05 & Rf4 \\
\hline
\end{tabular}

Note: 1) Allele is presented with the format of 'major allele / minor allele'

2) $M A F$ minor allele frequency

3) PVE phenotypic variation explained by each locus 


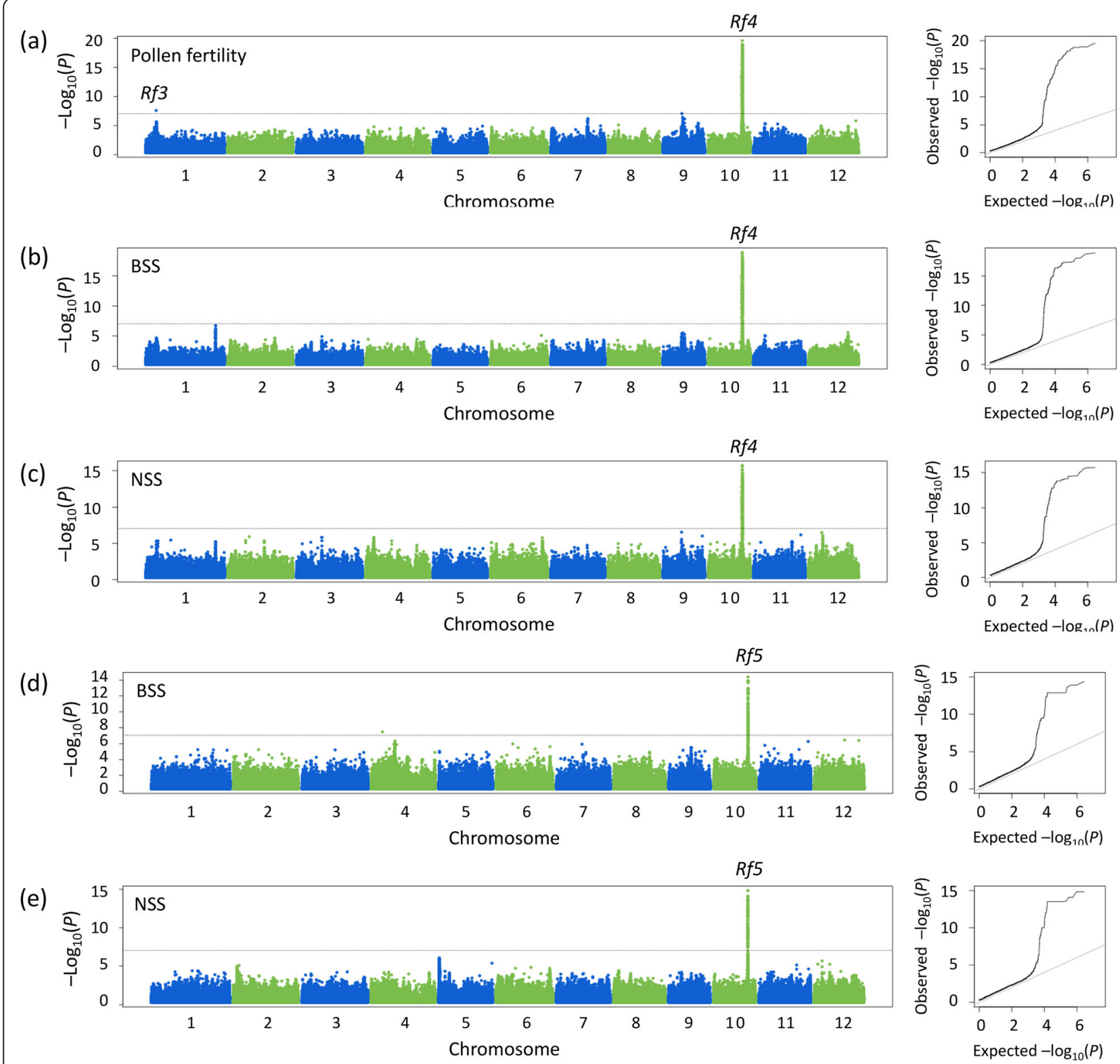

Fig. 4 Manhattan plots and quantile-quantile plots of pollen fertility (a), BSS (b) and NSS (c) of the $F_{1}$ population with the background of CMSWA, and BSS (d) and NSS (e) of the $F_{1}$ population with the background of CMS-HL, in year 2014. Negative $\log _{10}$-transformed $P$ values from a genome-wide scan are plotted against position on each of 12 chromosomes. Black horizontal dashed line indicates the genome-wide significance threshold

type is represented by the allele from the well-known restorer Minghui 63 (C147), and is evenly distributed in XI $I$ and $X I$ II accessions. Compared to $\mathrm{H} 1$, the $\mathrm{H} 2$ type shows two nonsynonymous SNPs and is mainly existed in XI II accessions. The H3 type shows 74 common SNPs which leads to a change of 50 amino-acid residues, and is mainly existed in aus accessions. The H4 type is represented by the allele from HUA and many wellknown maintainer lines such as Zhenshan 97B (C145), and shows 90 common SNPs and 2 large insertions, of which the first $1515 \mathrm{bp}$ insertion introduces a stop codon. The H4 type is mainly existed in XI I accessions.
The H5 type is only carried by two XI I accessions, and represented by the allele from Nipponbare, a geng/japonica accession having the reference genome of Oryza sativa. Compared to $\mathrm{H} 1, \mathrm{H} 5$ shows 62 common SNPs that leads to a change of 37 amino-acid residues. Results of multiple comparisons showed that both the values of pollen fertility and NSS of $\mathrm{H} 1$ and $\mathrm{H} 2$ were significantly higher than that of H3 and H4 in year 2013 and 2014 (Fig. 5b, Additional file 1: Figure S4a).

For $R f 5$, two main haplotypes and several rare haplotypes were classified according to sequence variations in coding region (Fig. 5c, Additional file 2: Table S4). The 
Table 2 Genome-wide significant associations for BSS and NSS of the $F_{1}$ population with the background of CMS-HL in year 2013 and 2014 using the linear mixed model

\begin{tabular}{|c|c|c|c|c|c|c|c|c|c|c|c|c|}
\hline \multirow[t]{2}{*}{ Trait } & \multirow[t]{2}{*}{ Chr. } & \multicolumn{5}{|l|}{2013} & \multicolumn{5}{|l|}{2014} & \multirow{2}{*}{$\begin{array}{l}\text { Known } \\
\text { loci }\end{array}$} \\
\hline & & Position & P-LMM & Allele $^{1)}$ & $\mathrm{MAF}^{2)}$ & PVE $(\%)^{3)}$ & Position & P-LMM & Allele & MAF & $\overline{\text { PVE }(\%)}$ & \\
\hline \multirow[t]{5}{*}{ BSS } & 2 & & & & & & $26,741,479$ & $2.24 \mathrm{E}-07$ & $\mathrm{G} / \mathrm{A}$ & 0.059 & 8.18 & \\
\hline & 4 & & & & & & $12,956,620$ & 1.17E-08 & $\mathrm{T} / \mathrm{C}$ & 0.359 & 8.81 & \\
\hline & 6 & & & & & & $21,359,687$ & 1.36E-07 & $\mathrm{A} / \mathrm{T}$ & 0.377 & 11.25 & \\
\hline & 10 & $18,828,056$ & 1.39E-08 & $\mathrm{G} / \mathrm{A}$ & 0.492 & 12.79 & $18,880,986$ & $1.74 \mathrm{E}-10$ & $A / G$ & 0.456 & 18.09 & Rf5 \\
\hline & 12 & & & & & & $16,744,603$ & 7.69E-08 & $\mathrm{A} / \mathrm{G}$ & 0.101 & 6.35 & \\
\hline NSS & 10 & $18,804,231$ & $4.08 \mathrm{E}-15$ & $\mathrm{~A} / \mathrm{C}$ & 0.231 & 28.25 & $18,876,148$ & 8.10E-20 & $\mathrm{A} / \mathrm{G}$ & 0.129 & 31.08 & Rf5 \\
\hline
\end{tabular}

Note: 1) Allele is presented with the format of 'major allele / minor allele'

2) $M A F$ minor allele frequency

3) PVE phenotypic variation explained by each locus

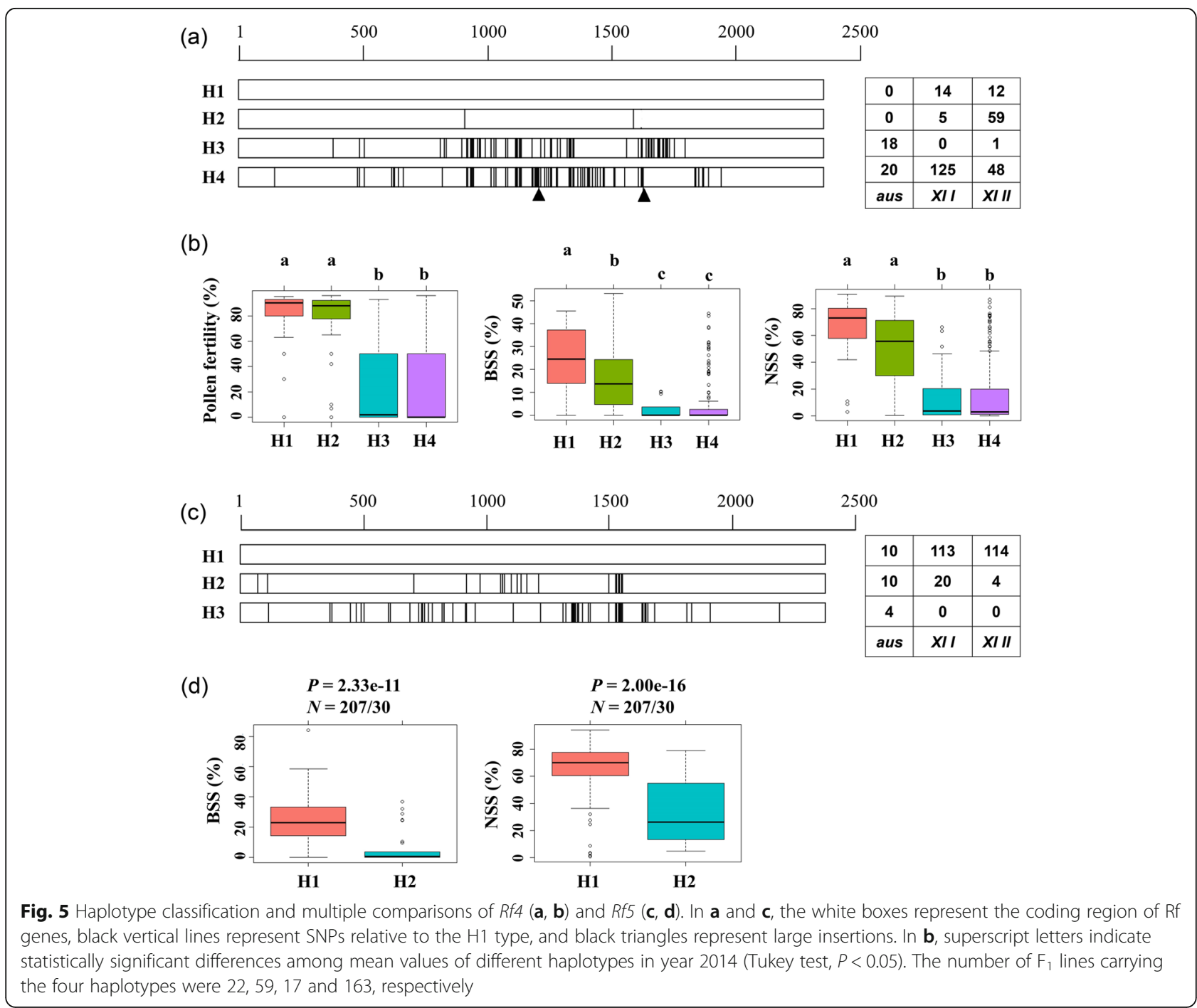


H1 type is represented by the allele from the well-known restorer 9311, while the $\mathrm{H} 2$ type is represented by the allele from YTA. Compared to $\mathrm{H} 1$, the $\mathrm{H} 2$ type shows 20 SNPs which leads to a change of 14 amino-acid residues, and the rare H3 type shows 60 SNPs including 39 nonsynonymous SNPs and is existed only in aus accessions. Results of multiple comparisons showed that both the values of BSS and NSS of $\mathrm{H} 1$ were significantly higher than that of H2 in year 2013 and 2014 (Fig. 5d, Additional file 1: Figure S4b).

For $R f 6$, eight haplotypes were identified according to sequence variations in genomic region (Fig. 6a). The H1 type is represented by the allele from 9311 , while $\mathrm{H} 4$ is represented by the allele from YTA. The first three types carry a $327 \mathrm{bp}$ insertion, among which the H1 type is carried by 36 out of 39 lines. Among the remaining five types that not carrying the insertion, $\mathrm{H} 4$ and $\mathrm{H} 6$ are mainly existed in XI I accessions, H5 is mainly in aus accessions, and H8 is mainly in XI $I$ accessions. With $R f 5$ fixed as the $\mathrm{H} 1$ type, multiple comparison of the six main Rf6 haplotypes revealed that no significant difference in BSS and NSS was observed among different types, except for between H5 and H6 (Fig. 6b).

\section{Discussion}

Genetic Basis Underlying Fertility Restoration of CMS-WA and CMS-HL

In this study, GWAS revealed that fertility restoration of CMS-WA was mainly conditioned by the major gene $R f 4$, as the remaining loci could not be repeatedly detected in 2 years and accounted for less variation (Table 1). The locus around $5.6 \mathrm{Mb}$ of chromosome 1 contributed $19.01 \%$ of the variation of pollen fertility in year 2014, and was located to the mapping region of $R f 3$ (Qi et al. 2008, Suresh et al. 2012, Yao et al. 1997, Zhang et al. 1997). The other loci were novel. For CMS-HL, Rf5 was the unique major gene in the association population, and the remaining loci were only responsible for BSS in 2014 (Fig. 4 , Table 2).

The majority of loci could not be repeatedly detected in 2 years, which may be attributed to two reasons. First of all, the number of loci conferring BSS far overweighs that conferring pollen fertility and NSS (Tables 1 and 2), demonstrating that some loci for BSS may be false, due to artificial and environmental effect. At flowering stage, selected panicles were tightly bagged, which affected the

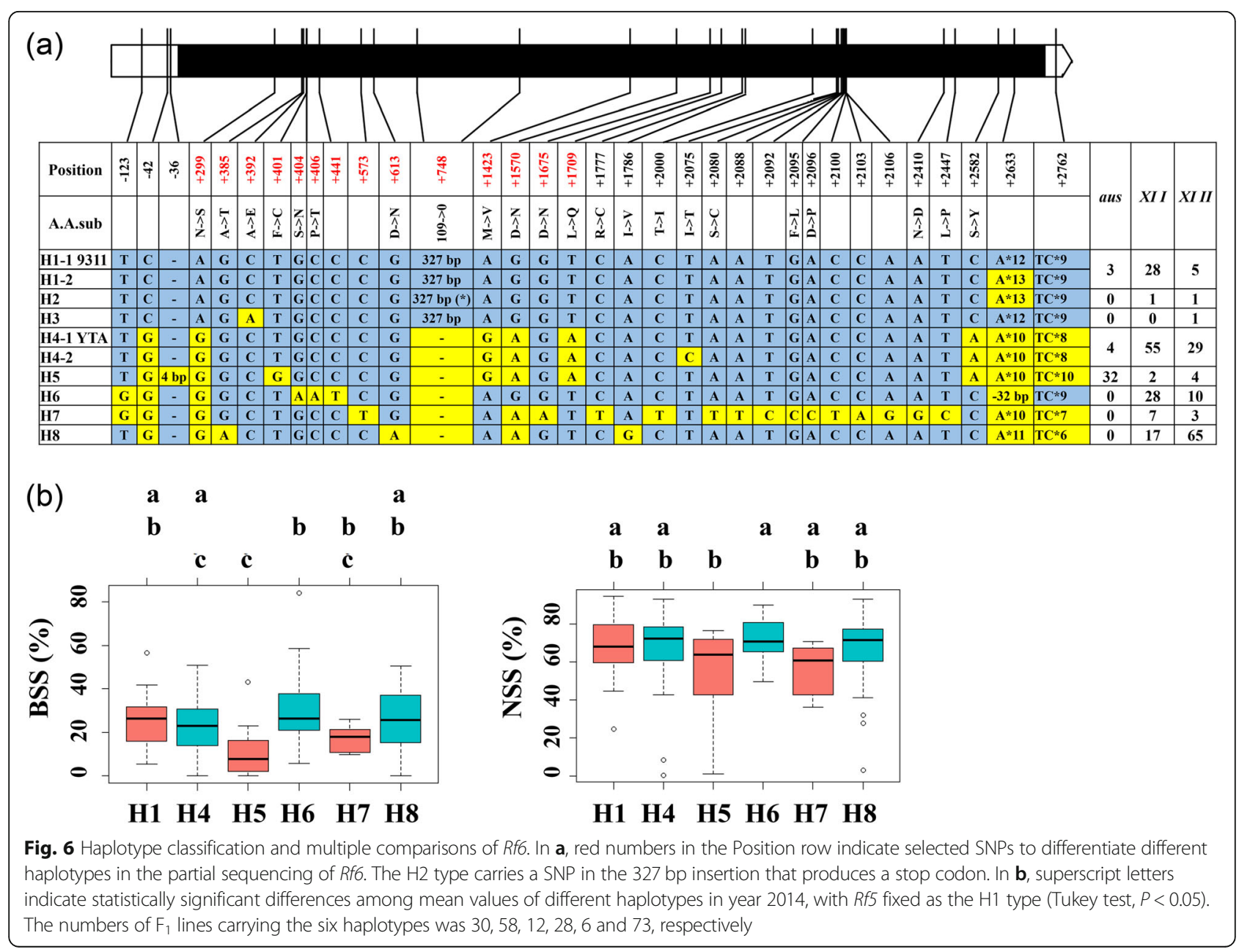


elongation of stem of different lines to varying degrees. Stems of some lines were bent over a large angle and even broken off, leading to less or no seed-setting rate. On the other hand, the bag limited the space of panicles, especially for those lines displaying large panicles, and caused increase in local temperature, which would also decrease seed-setting rate. In addition, the reality of the loci for BSS await further validation. Secondly, the size of the two association populations in 2013 was about 100 lines less than that in 2014 respectively, which could explain why some loci in 2014 were not detected in 2013, such as $R f 3$, the reported major gene for CMSWA (Additional file 2: Table S1). The 337 paternal accessions and the two maternal parents displayed huge variations in heading date (http://ricevarmap.ncpgr.cn/ v2/), which made it of great difficulty to make hybrids covering all paternal accessions in the planting season of year 2012, and some hybrids were further made in year 2013. With the two reasons above, the stable detection of $R f 4$ and $R f 5$ further demonstrated that the two are major genes for corresponding CMSs.

\section{Haplotype Analysis of $R f$ Genes}

Haplotype analysis of $R f 4$ revealed five types, among which the $\mathrm{H} 1, \mathrm{H} 2, \mathrm{H} 4$ and $\mathrm{H} 5$ had been reported by Tang et al. (2014). The H2 type is carried by the restorer IR24 (not in our accessions), demonstrating that it is functional, which is consistent with the result of multiple comparisons of fertility-related traits in our study (Tang et al. 2014). Therefore, the two nonsynonymous SNPs in $\mathrm{H} 2$ are likely to make little change to the function of RF4. No difference was observed between the three traits of $\mathrm{H} 3$ and $\mathrm{H} 4$, indicating that $\mathrm{H} 3$ is also a nonfunctional type, duo to the change of 50 amino-acid residues (Fig. $5 \mathrm{a}-\mathrm{b}$, Additional file 1: Figure S4a). In addition, among the paternal parents used in this study, aus accessions carry only the nonfunctional $\mathrm{H} 3$ and $\mathrm{H} 4$ type, 125 of $143 X I I$ accessions carry the nonfunctional $\mathrm{H} 4$ type, and 59 of 64 accessions carrying the functional $\mathrm{H} 2$ type belong to XI II group. The subgroup preferences of different $R f 4$ haplotypes implied that $R f 4$ had been subjected to selection in rice breeding, and contributed greatly to the differentiation of the three subgroups.

Haplotype analysis of $R f 5$ revealed two main haplotypes and several rare haplotypes that are carried by less than five accessions (Additional file 2: Table S1, Fig. 5c). The non-function $\mathrm{H} 2$ type carried by YTA does not have the functional SNP reported by $\mathrm{Hu}$ et al. (2012) (Additional file 2: Table S4). Therefore, the change of 14 amino-acid residues is likely to abolish the function of RF5 in fertility restoration, which is the same as the $\mathrm{H} 3$ type of $R f 4$. H1 type is carried by the majority of paternal accessions, implying that it has other important functions that facilitates its spreading in cultivated rice, though it is still not clear. Several rare haplotypes including the $\mathrm{H} 3$ type are existed only in aus accessions, and the two main haplotypes are also existed in aus accessions, showing that aus accessions are valuable germplasm resource for investigation of the evolution of $R f 5$ alleles (Additional file 2: Table S1).

Haplotype analysis of $R f 6$ revealed eight haplotypes, among which the first three types carry the functional $327 \mathrm{bp}$ insertion reported by Huang et al. (2015) (Fig. 6a). However, multiple comparison of the six main haplotypes indicated that the 327 insertion is not likely to be the functional variation. As BSS and NSS are only indirect reflections of fertility restoration, the conclusion above is awaited further to be validated with pollen fertility, which was unfortunately difficult to evaluate in this study.

\section{Difficulties in Mining $R f$ Genes Using GWAS}

GWAS have been proved to be powerful in genetic dissection of complex quantitative traits in rice and identifying candidate genes underlying target traits (Han and Huang 2013). However, its power suffered a major setback in mining $R f$ genes, at least in this study. GWAS of fertility-related traits revealed that both the fertility restoration of CMS-WA and CMS-HL were controlled by a major locus and several minor loci (Tables 1 and 2). However, the two major loci were located to a region containing about 10 genes encoding PPR proteins, which show high sequence homology (Tang et al. 2014). Without previous studies on gene cloning of Rf4 and Rf5, it would be of great difficulty to ascertain underlying functional genes. Furthermore, although the $R f 3$ region was detected for pollen fertility, the region about $\pm 100 \mathrm{~kb}$ away from the strongest signal contains 20 annotated ORFs, but none encode PPR proteins or other known homology proteins involved in fertility restoration, making it difficult to select candidate genes. Therefore, just like all the seven cloned $R f$ genes in rice viz $R f 1 a, R f 1 b$, $R f 2, R f 4, R f 5, R f 6$ and $R f 17$, map-based cloning is the only choice to narrow the target locus down to the smallest region containing the functional gene (Fujii and Toriyama 2009, Hu et al. 2012, Huang et al. 2015, Itabashi et al. 2011, Tang et al. 2014, Wang et al. 2006). In addition, the three fertility-related traits are easily affected by environment. The durable high temperature and humidity in Wuhan during the growing season exerted great pressure to the fate of developing and developed pollens, which resulted in the unrepeatable detection of many association signals in two different years, especially for those minor loci (Tables 1 and 2). Therefore, a combination of GWAS and linkage mapping would be better in mining $R f$ genes, which would provide not only an overview of the genetic basis, but 
also a high resolution of functional genes (Deng et al. 2017, Wang et al. 2018).

\section{Application in Development of Three-Line Hybrid Rice}

A three-line hybrid combination consists of three lines, a restorer, a CMS line and its maintainer line. No $R f$ genes are allowed in the genome of CMS lines and its maintainer lines, in order to maintain the complete sterility of CMS lines. In contrast, $R f$ genes are favored by restorers to restore the fertility of CMS lines as much as possible. Therefore, selection of $R f$ genes is of great importance in development of threeline hybrid rice. Some markers have been developed for $R f 4$ in previous studies (Chen et al. 2017, Suresh et al. 2012, Tang et al. 2014). In this study, the haplotypes of three $R f$ genes viz $R f 4, R f 5$ and $R f 6$ have been systematically classified using 337 accessions that covering the majority variation of $X I$ and aus accessions worldwide, providing valuable sequence variations for the development of co-segregating markers (Additional file 2: Table S3-S4, Fig. 6a). Take Rf4 for example. SNPs at the position of $+503,+919,+929 / 930,+1607,+1618,+$ 1621 of the coding region and the $1515 \mathrm{bp}$ insertion are cosegregated with the function of $R f 4$, and thus could be developed into suitable molecular markers to facilitate selection of Rft.

Except for major genes, minor $R f$ genes are vital in breeding process, which not only affect the sterility stability of CMS lines greatly, but also the degree of fertility restoration of restorers. However, the selection efficiency of minor $R f$ genes is far from expectations, due to the inability to precisely mapping them. In order to avoid the disturbance of minor $R f$ genes, new maintainer lines are always developed from progenies of existing maintainer lines, and so do restorers, which severely limit the genetic diversity of hybrid combinations. In this study, the ability of fertility restoration of 337 accessions for CMSWA and CMS-HL has been evaluated individually (Additional file 2: Table S1). The accessions that displayed no fertility restoration under the background of CMS could be directly used as maintainer lines or used as parents of novel maintainer lines, and those showing high fertility restoration under the background of CMS could be used in breeding of restorers. The majority of 337 accessions are inbred lines or landraces from worldwide, and several breeders are not familiar with them, suggesting that these accessions have not been exploited in hybrid rice breeding (personal communication, Xie et al. 2015). Therefore, results in this study could provide valuable germplasm resources to broaden the genetic diversity of three-line hybrid rice.

\section{Materials and Methods}

\section{Population Construction and Planting}

Two representative $X I$ CMS lines were used as maternal parents in this study, which were Hua1517A (HUA) and
YuetaiA (YTA). HUA is a CMS-WA line bred by our lab and shows high resistance to rice blast. YTA is a leading CMS-HL line, and its derived combination YTA/9311 displayed good performance in Southeast Asia countries (Zhu et al. 2010). The paternal parent population consisted of $337 \mathrm{XI}$ and aus lines from the 533 Oryza sativa germplasm accessions stored in our lab (Chen et al. 2014, Zhou et al. 2017). The $X I$ lines were further divided into two groups, $X I I$ and $X I I I$, as described in Zhou et al. (2017). The majority of XI I lines has germplasm of South China origin, while almost all of XI II lines are from IRRI or have parentage of IRRI varieties (Xie et al. 2015). Information about the accessions including names, countries of origin, geographical location, and subpopulation classification is listed in Additional file 1: Table S1.

Crosses were made between each line of the paternal parent population and the two maternal parents individually, and two $F_{1}$ populations with CMS-WA and CMS-HL background respectively were produced (Additional file 1: Figure $\mathrm{S} 1$ ). The two $\mathrm{F}_{1}$ populations and maternal parents were grown in a completely randomized design at the experimental farm of Huazhong Agricultural University in Wuhan, Hubei, during 2013 and 2014 growing seasons. Six plants per line were transplanted in a row with $16.5 \mathrm{~cm}$ between plants and 20.0 $\mathrm{cm}$ between rows. The 2 years were treated as two replications. Field management followed standard agricultural practice.

\section{Fertility Evaluation}

Three traits were used to evaluate the fertility of each $F_{1}$ line, which were pollen fertility, seed-setting rate of bagged panicles (BSS) and seed-setting rate of natural panicles (NSS).

Pollen fertility evaluation: At the flowering stage, five glumes with mature anther were randomly sampled from each plant and five plants from each line. Pollen grains from each plant were mixed, stained with $1 \% \mathrm{I}_{2}$ KI solution, and observed under an optical microscope. The ratio of dark-blue (stainable) pollen grains to total pollen grains was counted for each plant, and the average value of five plants of each line was termed as the pollen fertility of each line.

Seed-setting rate evaluation: At the flowering stage, five plants from each line and a panicle from each plant were bagged. At the maturity stage, the ratio of seedsetting glumes to total glumes on the bagged panicle was counted, and the average value of five plants of each line was termed as the BSS of each line. Similarly, the ratio of seed-setting glumes to total glumes on a natural panicle was counted, and the average value of five plants of each line was termed as the NSS of each line. 


\section{Genome-Wide Association Analysis}

SNP data for the 337 Oryza sativa accessions was reported in a previous study (Zhao et al. 2015) and the data is available at RiceVarMap v2.0 (http://ricevarmap. ncpgr.cn/v2/). Only SNPs with a minor allele frequency (MAF) $>5 \%$ and a missing rate $<20 \%$ were selected for association analysis. Finally, 2.7 million SNPs were used for GWAS.

GWAS on pollen fertility, BSS and NSS were respectively performed on the entire population using linear mixed models, as described in Zhou et al. (2017). The calculated genome-wide significance threshold was $P=8.7 \times$ $10^{-8}$, based on a nominal level of 0.05 . Phenotypic variation of each trait explained by multiple SNPs was calculated using R package "MLMM" (Segura et al. 2012). The physical locations of SNPs were identified based on the Rice Annotation version of 7.0 of variety Nipponbare from Michigan State University (http://rice.plantbiology. msu.edu/cgi-bin/gbrowse/rice/). Considering that the LD decay distance in $X I$ accessions is about $100 \mathrm{~kb}$ (Zhou et al. 2017), significant SNPs located to a region of less than $100 \mathrm{~kb}$ were treated as one locus.

\section{Haplotype Analysis}

For the three well-known genes conferring fertility restoration of CMS-WA or CMS-HL, Rf4, Rf5 and Rf6, a pair of primers were designed to amplify the full-length ORF individually. For each CMS, 50 representative paternal accessions were selected according to the three fertility-related traits, and the full-length ORF of the corresponding $R f$ gene was amplified using the high-fidelity DNA polymerase KOD FX (https://www.toyobo-global. $\mathrm{com} /$ ) and subjected to Sanger sequencing. The fulllength sequence of each gene was assembled from sequence reads using the software LaserGene (https:// www.dnastar.com/software/lasergene/). Primary haplotype classification of each gene was conducted with results of multiple sequence alignment using the software MEGA 7 (Kumar et al. 2016). According to the results of primary haplotype classification of each gene, the region covering rich SNPs to differentiate different haplotypes was identified, and a pair of primers were developed to amplify and sequence the target region of the remaining paternal accessions. The haplotypes of every gene were further classified based on the primary results of 50 accessions and the following sequencing results of the remaining accessions.

The haplotypes of each gene were displayed using software IBS1.1 (Fig. 5, Liu et al. 2015). All the primers used are listed in Additional file 2: Table S2.

\section{Statistical Analysis}

Pearson's correlation coefficients among fertility-related traits in each $F_{1}$ population were calculated with a two- sided $t$-test using cor function in $\mathrm{R}$, and displayed using the R package "corrplot". Variance analyses and multiple comparisons of the effects of different haplotypes of genes were computed using the R package "multcomp" with the method of Tukey test.

\section{Conclusions}

In this study, our results demonstrated that $R f 4$ and $R f 5$ are the two major genes for fertility restoration of CMSWA and CMS-HL respectively in the $X I$ accessions of rice. Haplotype analysis revealed that four main haplotypes for Rf4 display different subgroup preferences, and the functional type of $R f 5$ is carried by the majority of paternal accessions. Sequence variations of $R f 4, R f 5$ and $R f 6$ identified in this study could be of great use in marker-aided selection of corresponding genes in rice breeding. Besides, fertility evaluation of 337 accessions under the background of CMS could provide material resources for development of maintainer lines and restorers.

\section{Supplementary information}

Supplementary information accompanies this paper at https://doi.org/10. 1186/s12284-020-0372-0.

Additional file 1: Figure S1. The schematic of experimental design of our study. Figure S2. Distribution of pollen fertility (a), BSS (b) and NSS (c) of the F1 population with the background of CMS-WA, and BSS (d) and NSS (e) of the F1 population with the background of CMS-HL, in year 2013. Figure S3. Manhattan plots and quantile-quantile plots of pollen fertility (a), BSS (b) and NSS (c) of the F1 population with the background of CMS-WA, and BSS (d) and NSS (e) of the F1 population with the background of CMS-HL, in year 2013. Negative log10-transformed $P$ values from a genome-wide scan are plotted against position on each of 12 chromosomes. Black horizontal dashed line indicates the genome-wide significance threshold. Figure S4. Multiple comparisons of Rf4 (a) and Rf5 (b) using the phenotypic values in year 2013. In (a), superscript letters indicate statistically significant differences among mean values of different haplotypes (Tukey test, $P<0.05$ ). The number of $F 1$ lines carrying the four haplotypes was 10, 32, 7 and 88, respectively.

Additional file 2: Table S1. Names, origin, population structure, haplotypes of $R f$ genes and fertility traits of 337 O. sativa accessions. 'New' in the columns of the three $R f$ genes haplotype indicates that the paternal line carries a novel allele, which does not belong to any main haplotypes in this study. Table S2. Primers used in this study. Table S3. Haplotype classification of Rf4. Red numbers in the Position column indicate selected SNPs to differentiate different haplotypes in the partial sequencing of Rf4. Table S4. Haplotype classification of Rf5. Red numbers in the Position column indicate selected SNPs to differentiate different haplotypes in the partial sequencing of Rf5.

\section{Abbreviations}

BSS: Seed-setting rate of bagged panicles; CMS: Cytoplasmic male sterility; CMS-HL: Honglian CMS; CMS-WA: Wild-abortive CMS; GWAS: Genome wide association studies; HUA: Hua1517A; MAF: Minor allele frequency; NSS: Seedsetting rate of natural panicles; ORF: Open reading frame;

PPR: Pentatricopeptide repeat; Rf: Restorer of fertility; XI: Xian/indica; YTA: YuetaiA

\section{Acknowledgements}

We thank Professor Renshan Zhu from College of Life Science, Wuhan University and Professor Guo Xiaoling from College of the Environment \& Ecology, Xiamen University for providing us some rice materials. 


\section{Authors' Contributions}

PL performed most of experiments and wrote the paper. $\mathrm{HZ}$ and $\mathrm{RL}$ participated in data analysis. HY, DX, PS participated in phenotyping. QW and GW provided the genomic DNA of the paternal accessions. GG and QZ participated in field practice. YH designed and supervised this study. All authors read and approved the final manuscript.

\section{Funding}

This work was supported by grants from the National Program on R\&D of Transgenic Plants (2016ZX08001002-002), the Natural Science Foundation of China $(91935303,31821005)$ and the earmarked fund for the China Agriculture Research System (CARS-01-03).

\section{Availability of Data and Materials}

Genomic sequences have been deposited in the NCBI GenBank with accession numbers MN592683-MN592702 for different alleles of Rf4, MN592703-MN592706 for different alleles of Rf5, and MN592707-MN592716 for different alleles of Rf6.

The 337 accessions are available from Gongwei Wang on reasonable request. Hua1517A and YuetaiA are available from Yuqing He on reasonable request.

\section{Ethics Approval and Consent to Participate}

Not applicable.

\section{Consent for Publication}

Not applicable.

\section{Competing Interests}

The authors declare that they have no competing interests.

Received: 22 October 2019 Accepted: 28 January 2020

Published online: 10 February 2020

\section{References}

Bai XF, Zhao H, Huang Y et al (2016) Genome-wide association analysis reveals different genetic control in panicle architecture between indica and japonica rice. Plant Genome 9:2. https://doi.org/10.3835/plantgenome2015.11.0115

Bazrkar L, Ali AJ, Babaeian NA, Ebadi AA, Allahgholipour M, Kazemitabar K, Nematzadeh G (2008) Tagging of four fertility restorer loci for wild abortivecytoplasmic male sterility system in rice (Oryza sativa L.) using microsatellite markers. Euphytica 164:669-677. https://doi.org/10.1007/s10681-008-9667-8

Chen LK, Yan XC, Dai JH, Chen SP, Liu YZ, Wang H, Chen ZQ, Guo T (2017) Significant association of the novel Rf4-targeted SNP marker with the restorer for WA-CMS in different rice backgrounds and its utilization in molecular screening. J Integr Agr 16(10):2128-2135. https://doi.org/10.1016/S20953119(16)61620-9

Chen LT, Liu YG (2014) Male sterility and fertility restoration in crops. Annu Rev Plant Biol 65:579-606. https://doi.org/10.1146/annurev-arplant-050213-040119

Chen LT, Liu YG (2016) Discovery, utilization and molecular mechanisms of CMSWA in rice (in Chinese). Chin Sci Bull 61:3804-3812. https://doi.org/10.1360/ N972016-01044

Chen W, Gao YQ, Xie WB et al (2014) Genome-wide association analyses provide genetic and biochemical insights into natural variation in rice metabolism. Nat Genet 46:714-721. https://doi.org/10.1038/ng.3007

Deng M, Li DQ, Luo JY et al (2017) The genetic architecture of amino acids dissection by association and linkage analysis in maize. Plant Biotechnol J 15(10):1250-1263. https://doi.org/10.1111/pbi.12712

Dong HJ, Zhao H, Xie WB et al (2016) A novel tiller angle gene, TAC3, together with $T A C 1$ and $D 2$ largely determine the natural variation of tiller angle in rice cultivars. PLoS Genet 12(11):e1006412. https://doi.org/10.1371/journal. pgen. 1006412

Fujii S, Toriyama K (2009) Suppressed expression of RETROGRADE-REGULATED MALE STERILITY restores pollen fertility in cytoplasmic male sterile rice plants. Proc Natl Acad Sci U S A 106(23):9513-9518. https://doi.org/10.1073/pnas. 0901860106

Guo L, Qiu FL, Gandhi H, Kadaru S, De Asis EJ, Zhuang JY, Xie FM (2017) Genome-wide association study of outcrossing in cytoplasmic male sterile lines of rice. Sci Rep 7:3223. https://doi.org/10.1038/s41598-017-03358-9

Han B, Huang XH (2013) Sequencing-based genome-wide association study in rice. Curr Opin Plant Biol 16(2):133-138. https://doi.org/10.1016/j.pbi. 2013.03.006
Hu J, Wang K, Huang WC et al (2012) The rice pentatricopeptide repeat protein RF5 restores fertility in Hong-lian cytoplasmic male-sterile lines via a complex with the glycine-rich protein GRP162. Plant Cell 24:109-122. https://doi.org/ 10.1105/tpc.111.093211

Hu J, Zhu RS, Li SQ, Li YS, Yu JH, Huang WC, Zhu YG (2016) Discovery, utilization and perspective of Honglian cytoplasmic male sterile rice (in Chinese). Chin Sci Bull 61:3813-3821. https://doi.org/10.1360/N972016-01094

Huang WC, Hu J, Yu CC, Huang Q, Wan L, Wang LL, Qin XJ, Ji YX, Zhu RS, Li SQ, Zhu YG (2012a) Two non-allelic nuclear genes restore fertility in a gametophytic pattern and enhance abiotic stress tolerance in the hybrid rice plant. Theor Appl Genet 124:799-807. https://doi.org/10.1007/s00122-0111755-9

Huang WC, Yu CC, Hu J et al (2015) Pentatricopeptide-repeat family protein RF6 functions with hexokinase 6 to rescue rice cytoplasmic male sterility. Proc Natl Acad Sci U S A 112:14984-14989. https://doi.org/10.1073/pnas. 1511748112

Huang XH, Zhao Y, Wei XH et al (2012b) Genome-wide association study of flowering time and grain yield traits in a worldwide collection of rice germplasm. Nat Genet 44:32-39. https://doi.org/10.1038/ng.1018

Itabashi E, Iwata N, Fujii S, Kazama T, Toriyama K (2011) The fertility restorer gene, Rf2, for Lead Rice-type cytoplasmic male sterility of rice encodes a mitochondrial glycine-rich protein. Plant J 65:359-367. https://doi.org/10. 1111/j.1365-313X.2010.04427.x

Kazama T, Toriyama K (2014) A fertility restorer gene, Rf4, widely used for hybrid rice breeding encodes a pentatricopeptide repeat protein. Rice 7:28. https:// doi.org/10.1186/s12284-014-0028-z

Kumar S, Stecher G, Tamura K (2016) MEGA7: molecular evolutionary genetics analysis version 7.0 for bigger datasets. Mol Biol Evol 33:1870-1874. https:/ doi.org/10.1093/molbev/msw054

Li SQ, Yang DC, Zhu YG (2007) Characterization and use of male sterility in hybrid rice breeding. J Integr Plant Biol 49:791-804. https://doi.org/10.1111/j.17447909.2007.00513.x

Liu WZ, Xie YB, Ma JY et al (2015) IBS: an illustrator for the presentation and visualization of biological sequences. Bioinformatics 31:3359-3361. https:// doi.org/10.1093/bioinformatics/btv362

Luo DP, Xu H, Liu ZL et al (2013) A detrimental mitochondrial- nuclear interaction causes cytoplasmic male sterility in rice. Nat Genet 45:573-577. https://doi.org/10.1038/ng.2570

Peng X, Wang K, Hu C, Zhu Y, Wang T, Yang J, Tong J, Li S, Zhu Y (2010) The mitochondrial gene orfH79 plays a critical role in impairing both male gametophyte development and root growth in CMS-Honglian rice. BMC Plant Biol 10:125. https://doi.org/10.1186/1471-2229-10-125

Qi FL, Jiang MS, Yuan SJ, Yao FY, Gao J, Li GX (2008) Mapping of fertility-restoring gene Rf3 for wild-abortive cytoplasmic male sterility in rice (in Chinese). Chin Agric Sci Bull 24(8):114-117

Qin XJ, Huang Q, Xiao HJ, Zhang QN, Ni CZ, Xu YH, Liu G, Yang DC, Zhu YG, Hu J (2016) The rice DUF1620-containing and WD40-like repeat protein is required for the assembly of the restoration of fertility complex. New Phytol 210:934945. https://doi.org/10.1111/nph.13824

Segura V, Vilhjálmsson BJ, Platt A, Korte A, Seren Ü, Long Q, Nordborg M (2012) An efficient multi-locus mixed-model approach for genome-wide association studies in structured populations. Nat Genet 44:825-830. https://doi.org/10. 1038/ng.2314

Suresh PB, Srikanth B, Kishore VH et al (2012) Fine mapping of Rf3 and Rf4 fertility restorer loci of WA-CMS of rice (Oryza sativa L.) and validation of the developed marker system for identification of restorer lines. Euphytica 187(3): 421-435. https://doi.org/10.1007/s10681-012-0737-6

Tang HW, Luo DP, Zhou DG, Zhang QY, Tian DS, Zheng XM, Chen LT, Liu YG (2014) The rice restorer Rf4 for wild-abortive cytoplasmic male sterility encodes a mitochondrial-localized PPR protein that functions in reduction of WA352 transcripts. Mol Plant 7:1497-1500. https://doi.org/10.1093/mp/ssu047

Wang H, Xu ST, Fan YM et al (2018) Beyond pathways: genetic dissection of tocopherol content in maize kernels by combing linkage and association analyses. Plant Biotechnol J 16(8):1464-1475. https://doi.org/10.1111/pbi. 12889

Wang K, Gao F, Ji YX, Liu Y, Dan ZW, Yang PF, Zhu YG, Li SQ (2013) ORFH79 impairs mitochondrial function via interaction with a subunit of electron transport chain complex III in Honglian cytoplasmic male sterile rice. New Phytol 98:408-418. https://doi.org/10.1111/nph.12180

Wang ZH, Zou YJ, Li XY et al (2006) Cytoplasmic male sterility of rice with boro II cytoplasm is caused by a cytotoxic peptide and is restored by two related 
PPR motif genes via distinct modes of mRNA silencing. Plant Cell 18:676687. https://doi.org/10.1105/tpc.105.038240

Xie WB, Wang GW, Yuan M et al (2015) Breeding signatures of rice improvement revealed by a genomic variation map from a large germplasm collection. Proc Natl Acad Sci U S A 112(39):E5411-E5419. https://doi.org/10.1073/pnas. 1515919112

Yao FY, Xu CG, Yu SB, Li JX, Gao YJ, Li XH, Zhang QF (1997) Mapping and genetic analysis of two fertility restorer loci in the wild-abortive cytoplasmic male sterility system of rice (Oryza sativa L.). Euphytica 98:183-187. https://doi.org/ 10.1023/A:1003165116059

Zhang GQ, Lu YG, Bharaj TS, Virmani SS, Huang N (1997) Mapping of the Rf3 fertility-restoring gene for WA cytoplasmic male sterility in rice using RAPD and RFLP markers. Theor Appl Genet 94:27-33. https://doi.org/10.1007/ s001220050377

Zhao H, Yao W, Ouyang YD, Yang WN, Wang GW, Lian XM, Xing YZ, Chen LL, Xie WB (2015) RiceVarMap: a comprehensive database of rice genomic variations. Nucleic Acids Res 43:D1018-D1022. https://doi.org/10.1093/nar/gku894

Zhou H, Li PB, Xie WB et al (2017) Genome-wide association analyses reveal the genetic basis of stigma exsertion in rice. Mol Plant 10:634-664. https://doi. org/10.1016/j.molp.2017.01.001

Zhu RS, Yu JH, Ding JP et al (2010) Research and utilization of honglian type hybrid rice (in Chinese). Hybrid Rice 25(S1):12-16

Zhuang JY, Fan YY, Wu JL, Rao ZM, Xia YW, Zheng KL (2001) Mapping genes for rice CMS-WA fertility restoration (in Chinese). J Genet Genomics 28(2):129-134

\section{Publisher's Note}

Springer Nature remains neutral with regard to jurisdictional claims in published maps and institutional affiliations.

\section{Submit your manuscript to a SpringerOpen ${ }^{\circ}$ journal and benefit from:}

- Convenient online submission

- Rigorous peer review

- Open access: articles freely available online

High visibility within the field

- Retaining the copyright to your article

Submit your next manuscript at $\boldsymbol{\nabla}$ springeropen.com 